\title{
Juegos digitales y gamificación aplicados en el ámbito de la educación
}

\section{Digital games and gamification applied to education}

\author{
Ruth S. Contreras Espinosa
}

Universitat de Vic-Universitat Central de Catalunya (España)

Coordinadora del Monográfico

\section{JUEGOS DIGITALES, GAMIFICACIÓN Y APRENDIZAJE}

Durante mucho tiempo dio la impresión de que los juegos eran la antítesis del aprendizaje. Sin embargo, la opinión popular ha cambiado durante los últimos años mostrando la conexión que existe entre los juegos y el aprendizaje. Durante la última década ambos han estado conectados mucho más de lo que parece. Los juegos ayudan a experimentar con nuevas identidades, a explorar opciones y consecuencias, y a probar nuestros propios límites. Mediante los juegos es posible el desarrollo de habilidades sociales (Perrota et al., 2013), la motivación hacia el aprendizaje (Kenny y McDaniel, 2011), una mejora en la atención, la concentración, el pensamiento complejo y la planificación estratégica (Kirriemuir y McFarlane, 2004). Incluso ayudan a interiorizar conocimientos multidisciplinarios (Mitchell y Savill-Smith, 2004), propician un pensamiento lógico y crítico y a mejorar habilidades que ayudan a resolver diversos problemas (Higgins et al., 1999), desarrollar habilidades cognitivas y a la toma de decisiones técnicas (Bonk y Dennen, 2005). El profesor puede desplegar diferentes estrategias orientadas a potenciar el conocimiento de hechos y datos concretos gracias a los contenidos educativos presentes en el juego como, por ejemplo, conocer la vida de un personaje y su actividad o el conocimiento condicional (Paris et al., 1983), utilizando conocimientos adquiridos previamente, para plantear estrategias de forma consciente.

Por supuesto que existen límites en lo que puede hacer cualquier juego, al igual que con cualquier programa educativo, proceso o actividad. Un juego puede encajar en particular con una necesidad pedagógica específica, un público en concreto, en un conjunto de objetivos y limitaciones y, al mismo tiempo, ese juego podría no ser apropiado en un contexto diferente. Un juego puede apoyar a ciertos estilos de aprendizaje o necesidades de cualificación, pero no a todos. Del mismo modo que se mencionan sus posibilidades, los límites también deberían de ser identificados. Necesitamos entender no sólo si un juego digital o una estrategia de gamificación puede enseñar, sino además las condiciones en las que puede -o no- ayudar a alguien a aprender. 
Aunque el término gamification se ha extendido y nos hace hablar del poder de utilizar elementos del juego y el diseño de juegos, para mejorar el compromiso y la motivación de los participantes -el uso de elementos de juego en contextos que no son de juego-, lo que muchos profesionales, profesores e investigadores han estado haciendo es diseñar experiencias atractivas, igual que en un videojuego. Pero el concepto gamification es diferente a serious game o a game-based learning, mientras que el segundo describe el diseño de juegos que no tienen el firme objetivo de entretener a los usuarios, las experiencias "gamificadas" simplemente emplean algunos elementos de los juegos como reglas, mecánicas, etc. Game-based learning o aprendizaje basado en juegos, por su parte y en términos generales, se refiere al uso de juegos para apoyar al proceso de enseñanza/aprendizaje. Los principios fundamentales así como los mecanismos involucrados en el proceso se centran en lograr unos resultados de aprendizaje específicos, pero de manera colateral también se logran resultados secundarios, como por ejemplo, un juego que tiene como objetivo la enseñanza del idioma inglés también puede conducir a una actitud positiva hacia la asignatura de lengua. La motivación es necesaria para garantizar el aprendizaje y para evaluar un programa educativo pues cuando un estudiante se encuentra motivado, la efectividad de la actividad aumenta. En teoría, la investigación longitudinal relacionada con el aprendizaje basado en juegos ofrece la posibilidad de variar el contexto, las variables de intermediación, mejorar la motivación de los estudiantes, así como la experiencia de los facilitadores del juego y, a la larga, una mejora en las instalaciones institucionales (Kenny y McDaniel, 2011).

En los últimos años, la gamificación se ha visto envuelta en una rápida adopción de iniciativas ecológicas, de marketing, empresariales y por supuesto, en la educación. El uso de esas experiencias ha aumentado rápidamente y sugiere la necesidad de reevaluar la intersección entre juegos y aprendizaje.

Evaluar la eficacia de los juegos digitales o del uso de estrategias de gamificación ha demostrado ser todo un reto. La evaluación de otros tipos de intervenciones y estrategias educativas es una tarea que podría denominarse relativamente sencilla en comparación con el uso de juegos -sobre todo los digitales-. La tarea se complica debido a que es necesario evaluar diversos factores, ecologías y contextos. Pero a pesar de estos desafíos, en la última década la investigación también ha florecido exponencialmente en este sentido. Si observamos estudios como el de Mayer et al (2013) o revisiones de literatura como la de All et al. (2014), Perrotta et al. (2013) o Fui-Hoon Nah et al. (2014) donde se analizan diversos estudios relacionados con la eficacia y la evaluación, podremos ver claramente como decenas de autores se han centrado en evaluar su eficacia.

El uso de una metodología de evaluación común y estandarizada que ayude a evaluar todo un proceso de aprendizaje utilizando un juego digital no solo debería servir para estudiar y comparar lo que ocurre, sino también, debería de ser lo suficientemente flexible como para poder personalizarla y permitir ciertos ajustes en diversos contextos. Esto puede llegar a ser todo un desafío. Además, se debe tomar 
en cuenta que mientras se realizan los estudios de campo, tanto las preguntas de investigación como los métodos pueden tomar vida propia precisamente por las condiciones de cada caso. Sin embargo, las sugerencias de autores como Mayer et al. (2013) y Lieberoth y Roepstorff (2015) indican que lo mejor es centrarse en el uso de métodos mixtos como una opción que puede ser desplegada en diferentes etapas, incluyendo desde la recogida de datos hasta el análisis de los mismos. Esto incluye el uso de metodologías en donde, además, sea posible utilizar diferentes medidas objetivas de rendimiento. Obviamente el uso de los métodos dependerá del presupuesto de los investigadores pero en especial de la capacidad de cada uno para analizar los datos obtenidos.

A pesar de todas las innovaciones tecnológicas, sociales y económicas que nos han permitido crear, reproducir, replicar e investigar los juegos, no podemos pasar por alto las muchas formas que estos pueden adoptar. Los juegos, ya sean digitales, híbridos, analógicos virtuales, en línea, offline, de consola, para la Web o móviles, son juegos. Los seres humanos han estado jugando y aprendiendo de los juegos desde el inicio de la humanidad y no podemos olvidar que en su esencia más básica, nos ayudan a compartir y comunicar lo que somos. El juego es más viejo que la propia cultura y la razón por la cual puede ser culturalmente valioso es porque tiene una función en sí mismo. Por estas razones, es el momento apropiado para considerar esa conexión que existe entre los juegos y el aprendizaje.

\section{SOBRE EL NÚMERO ACTUAL DE RIED}

El presente monográfico, auspiciado con el epígrafe: "Juegos Digitales y Gamificación aplicados en el ámbito de la Educación”, trata de ofrecer diferentes perspectivas teóricas y prácticas que intentan facilitar la reflexión hacia estos fenómenos. En este sentido, los coordinadores del mismo ofrecen un pequeño grupo de estudios significativos que articulan los límites y potencialidades de los juegos digitales y de las estrategias de gamificación utilizadas para facilitar el aprendizaje. Se identifican algunas prácticas y casos que abren nuevas incógnitas y que exploran áreas que aún se deben examinar en profundidad.

En una primera instancia, los profesores Inés Susana Evaristo Chiyong, María Vanessa Vega Velarde y Ricardo Navarro Fernández, de Perú, esbozan de una forma acertada en su artículo: "Uso de un videojuego educativo como una herramienta para aprender la historia del Perú", la incorporación de juegos digitales como una estrategia diferente para el aprendizaje. En el crepúsculo del artículo se vislumbra que este tipo de juegos se pueden asumir como una tecnología más que los docentes disponen para crear una verdadera escenografía virtual de los hechos históricos relacionados con la independencia del Perú. Los resultados obtenidos en su estudio demuestran el efecto logrado posteriormente en las calificaciones de los estudiantes.

La profesora Patricia Margarida Farias Coelho, de la Universidade de Santo Amaro, y el profesor Marcos Rogério Martins Costa, de la Universidade de São Paulo, 
Brasil, en su artículo: "Uma ferramenta digital que faz games educativos: o contexto brasileiro de ensino e aprendizagem", señalan a la herramienta FazGame, utilizada en el proceso de creación de juegos en línea, como una propuesta innovadora en el escenario educativo brasileño, ya que el software permite la creación y publicación de juegos educativos sin necesidad de que los profesores y usuarios cuenten con conocimientos de diseño, género de juegos o programación. Este instrumento surge como fruto del trabajo de los investigadores Carla Zeltzer y Antônio Flávio Oliveira Ramos, bajo los auspicios de la marca FazGame. La evidencia presentada indica que la herramienta es apreciada por profesores de Brasil y se considera fácil de utilizar en la creación de juegos digitales.

$\mathrm{El}$ siguiente trabajo vinculado con entornos digitales ha sido realizado por los profesores Ruth S. Contreras Espinosa, José Luis Eguia y Lluis Solano Albajes con el título: "Investigación-acción como metodología para el diseño de un serious game“. Consiste en una investigación que describe una experiencia llevada a cabo por un grupo multidisciplinario de investigadores en colaboración con profesores de primaria de la ciudad de Barcelona para desarrollar un juego que permite trabajar competencias de educación orientadas a estudiantes de primaria. Los resultados analíticos muestran el diseño de un juego utilizando un proceso de cocreación con base en la metodología investigación-acción. El juego llamado "Ferran Alsina" se crea como un facilitador de una nueva metodología de enseñanza y se centra en trabajar con competencias básicas, metodológicas y específicas para niños con edades entre los 8 y 11 años de edad. Por último, manifiestan la necesidad de un mayor número de investigaciones claras que muestren experimentos y metodologías para el diseño de serious games.

João Mattar y Sérgio Nesteriuk de la Universidade Anhembi Morumbi, Brasil, continúan los discursos en su artículo "Estratégias do Design de Games que podem ser incorporadas à Educação a Distância” y nos explicitan que las teorías relacionadas con el aprendizaje basado en juegos, así como algunas estrategias de gamificación se pueden incorporar en la educación y en esto incluye a la educación a distancia. Su objetivo es proponer un modelo teórico basado en los tres niveles de investigación propuestos por Zawacki-Richter y Anderson (2015), que implican a modelos de investigación, tecnología e innovación y el diseño de la instrucción y la interacción. Se trata de una investigación bibliográfica, no empírica, que analiza los principios y elementos que conforman el diseño de juegos y que se pueden incorporar en el diseño educativo, identificando algunas categorías como: el aprendizaje lúdico, el juego para mantener al jugador constantemente motivado, el equilibrio para mantener al jugador en un estado de flujo, la personalización de la experiencia del jugador y la cocreación, entre otras. Se concluye que en la educación es necesario el uso de modelos flexibles centrados en la colaboración, interacción y en el aprendizaje.

En la misma línea discursiva, la profesora Eliane Schlemmer, de la Universidade do Vale do Rio dos Sinos, presenta otro artículo titulado: "Games e Gamificação em Espaços de Convivência Híbridos, Multimodais e Pervasivos: uma alternativa aos 
modelos de EaD”. En él muestra la necesidad de abordar estudios para entender el potencial de la hibridación, la multimodalidad y la omnipresencia, combinando juegos y gamificación como instrumentos que proporcionan experiencias y conocimiento. Su objetivo es comprender la contribución de los "Games e Gamificação" y mediante una investigación exploratoria y cualitativa, se inspira en el método cartográfico. Se hace uso de la observación, los registros de bitácora, audio, foto y vídeo digital. En conclusión, los resultados muestran que ambas propuestas permiten el desarrollo de situaciones de enseñanza y aprendizaje y que se configuran como elementos híbridos y multimodales. Además, se destaca que existe una mayor participación en sujetos en con formación inicial, en educación primaria, a partir de lecturas críticas que forman parte de su rutina escolar.

Cierra el monográfico el artículo realizado por Emiliano Labrador y Eva Villegas, con el título: "Unir Gamificación y Experiencia de Usuario para mejorar la experiencia docente". Los profesores de La Salle-Universitat Ramon Llull, en Barcelona, se centran en explicar una experiencia "gamificada" que ha sido diseñada y evaluada para motivar a sus estudiantes. Los participantes con un perfil de base principalmente tecnológico, respondieron a encuestas basadas en técnicas de evaluación de experiencia de usuario con el fin de proporcionar datos tanto de la aplicación del contenido académico como de los puntos positivos y negativos de las mecánicas de gamificación que fueron aplicadas en clase. Las encuestas se contrastaron entonces con los resultados académicos de los alumnos tanto en notas parciales como en sus notas finales. Asimismo, con respecto a los resultados obtenidos, el grupo de estudiantes del curso 2013-2014 mostró una mejoría.

\section{REFLEXIÓN FINAL}

Quisiera cerrar mi reflexión con las palabras de Johan Huizinga en su famoso libro Homo Ludens de 1983. Huizinga explica que jugar es un acto que constituye diversas formas culturales que facilitan las normas o códigos de la semiótica de la sociedad. El jugador se compromete a seguir unas normas que rigen su conducta y, de esta forma, el juego permite al sujeto realizar acciones que no tienen por qué tener consecuencias materiales:

Activity standing quite consciously outside 'ordinary' life as being 'not serious', but at the same time absorbing the player intensely and utterly. It is an activity connected with no material interest, and no profit can be gained by it... It promotes the formation of social groupings which tend to surround themselves with secrecy and to stress their difference from the common world by disguise or other means.

El juego es auto-generador y auto-motivador y no es un comportamiento empírico, sino más bien un determinado número de acciones. Se podría incluso ir más allá, proponiendo que el juego puede ser una actividad compartida entre 
personas que reconocen con entusiasmo una declinación inherente del sistema de juego. Esta declinación implica a la comunicación, la cual define el resultado del juego. El juego requiere además que el jugador tenga un cierto estado de ánimo -una predisposición a jugar- y que siga unas reglas, exactamente igual que pasa en la educación, al igual que con cualquier programa educativo, proceso o actividad. Juegos y aprendizaje, durante la última década han estado conectados mucho más de lo que parece ayudando a las personas a experimentar, a explorar y a probar nuestros propios límites. Necesitamos más teoría de manera que los términos y los principios asociados invoquen a lograr un nuevo contexto.

El presente número de RIED se completa con otros artículos miscelánea que, aunque no forman parte del monográfico “Juegos Digitales y Gamificación aplicados en el ámbito de la Educación", suponen aportaciones interesantes al campo de estudio propio de esta revista centrada en propuestas educativas de carácter no presencial.

\section{REFERENCIAS BIBLIOGRÁFICAS}

All, A., Núñez, P., y Van, J. (2014). Measuring effectiveness in Digital Game-Based Learning: A Methodological Review. International Journal of Serious Games, 1(2), 3-21.

Bonk, C., y Dennen, V. (2005). Massive multiplayer online gaming a research framework for military training and education. Washington, D.C.: Office of the Under Secretary of Defense (Personnel and Readiness), Readiness and Training Directorat, Advanced Distributed Learning (ADL) Initiative.

Fui-Hoon Nah, F., Zeng, Q., Rajasekhar, V., Padmanabhuni, A., y Eschenbrenner, B. (2014). Gamification of Education: A review of Literature. HCI in Business, 8527, 401-409.

Higgins, E., Grant, H., y Shah, J. (1999). Self Regulation and quality of life: Emotional and nonemotional life experiences. In D. Kahneman, E. Diener y N. Schwarz (Eds.), Well-being: The foundations of hedonic psychology, (244-266). Nueva York: Russell Sage Foundation.

Kenny, R., y McDaniel, R. (2011). The role teachers' expectations and value assessments of video games play in their adopting and integrating them into their classrooms. British Journal of Educational Technology, 42(2), 197-213.

Kirriemuir, J., y McFarlane, A. (2004). Literature review in games and learning. http://www.futurelab.org.uk/download/ pdfs/research/lit reviews/Games Review1

Lieberoth, A., y Roepstorff, A. (2015). Mixed methods in games research, playing on strengths and countering weaknesses. In P. Lankoski, B. Staffan. Game research methods: An overview, (271-289). Pittsburgh: ETC Press.

Mayer, I., Bekebrede, G., Harteveld, C., Warmelink, Q. Z., Van Ruijven, T., Lo, J., Kortmann, R., y Wenzler, I. (2013). The research and evaluation of serious games: Toward a comprehensive methodology. British Journal of Education Technology, 45(3), 502-527.

Mitchell, A., y Savill-Smith, C. (2004). The use of computer and video games for learning: A review of the literature. Londres: Learning and Skills Development Agency.

Paris, S., Lipson, M., y Wixson, K. (1983). Becoming a Strategic Reader. Contemporary Educational Psychology, 8, 293-316. 
Perrotta, C., Featherstone, G., Aston, H., y Houghton, E. (2013). Gamebased Learning: Latest Evidence and Future Directions. En NFER Research Programme: Innovation in Education. Slough- Berkshire. http://ocw.metu.edu.

tr/pluginfile.php/10919/mod resource content/1/GAME01.pdf

Zawacki-Richter, O., y Anderson, T. (2015). Educação a Distância Online: construindo uma agenda de pesquisa. São Paulo: Artesanato Educacional.

\section{PERFIL ACADÉMICO Y PROFESIONAL DE LA COORDINADORA}

Ruth S. Contreras-Espinosa. Doctora en Ingeniería Multimedia. Profesora de la Facultad de Empresa y Comunicación y de la Facultad de Ciencias y Tecnología en la Universitat de Vic-Universitat Central de Catalunya. Miembro del grupo Research group on Data and Signal Processing de la misma Universidad.

E-mail: ruth.contreras@uvic.cat

\section{DIRECCIÓN DE LA AUTORA}

Facultad de Empresa y Comunicación

Universitat de Vic-Universitat Central de Catalunya

Despacho Eoo1

C/Sagrada Familia 7, 08500, Vic

Fecha de recepción del artículo: 07/03/2016

Fecha de aceptación del artículo: 09/03/2016

\section{Como citar este artículo:}

Contreras-Espinosa, R. S. (2016). Presentación. Juegos digitales y gamificación aplicados en el ámbito de la educación. RIED. Revista Iberoamericana de Educación a Distancia, 19(2), pp. 27-33. doi: http://dx.doi.org/10.5944/ried.19.2.16143 